\title{
关于 SBK 算子对有界变差 函数的点态逼近度
}

\author{
郭 顺 生 \\ (河北师范大学数学系, 石家庄)
}

\section{一、引言}

Cheng 在文献[1]中研究了 Bernstein 算子

$$
B_{n}(f, x)=\sum_{k=0}^{n} f\left(\frac{k}{n}\right) p_{n k}(x), \quad(x \in[0,1])
$$

其中

$$
p_{n k}(x)=\left(\begin{array}{l}
n \\
k
\end{array}\right) x^{k}(1-x)^{n-k},
$$

对有界变差函数的点态逼近度. Sikkema 在文献[2]中引入算子(也可见文献[3] p. 173)

$$
C_{n}(f, x)=\sum_{k=0}^{n} f\left(\frac{k}{n+\alpha(n)}\right) p_{n k}(x),
$$

其中 $\alpha(n) \geqslant 0$ 且 $\lim _{n} \frac{\alpha(n)}{n}=0$. 显然, $B_{n}(f, x)$ 是 $C_{n}(f, x)$ 当 $\alpha(n)=0$ 时的特殊情况. Sikkema 研究了 $C_{n}(f, x)$ 对 $C[0,1]$ 中函数的收敛性质. 本文中, 我们限定

$$
\lim _{n} \frac{\alpha(n)}{\sqrt{n}}=0 \text {. }
$$

另外，我们引进如下算子:

$$
C_{n}^{*}(f, x)=\left(\varphi_{n}+1\right) \sum_{k=0}^{n} p_{n k}(x) \int_{\frac{k}{p_{n}+1}}^{\frac{k+1}{p_{n}+1}} f(t) d t,
$$

其中 $\varphi_{n}=n+\alpha(n), \alpha(n) \geqslant 0$ 且满足 (1.3) 式. 我们称 $C_{n}^{*}$ 为 Sikkema-Bernstein-Kantorovitch 算子(简称 SBK 算子). 显然, 当 $\alpha(n)=0$ 时,它即为 Bernstein-Kantorovitch 算子. 本 文利用概率论中的一些结果给出了 $C_{n}(f, x)$ 及 $C_{n}^{*}(f, x)$ 对 $[0,1]$ 上有界变差函数的收玫速 度. 作为本文结果的特例, 它改进了文献 [1]中的结果. 最后我们指出本文得到的逼近度是不 能改进的.

\section{二、引理}

首先引用概率中的一个结果(见文献 [4], p. 159).

本:文1985年11月26日收到。

第 20 期

科学通报 
引理 1 设独立随机变数列 $\left\{\xi_{k}\right\}$ 有相同的分布, 且 $0<D \xi_{k}<\infty, \beta_{3}=E\left|\xi_{r}-E \xi_{i}\right|^{3}<\infty$, 则

$$
\max _{y}\left|P\left(-\frac{1}{b_{1} \sqrt{n}} \sum_{k=1}^{n}\left(\xi_{k}-a_{1}\right) \leqslant y\right)-\frac{1}{\sqrt{2 \pi}} \int_{-\infty}^{y} e^{-\frac{z^{2}}{2}} d t\right|<-\frac{c}{\sqrt{n}} \frac{\beta_{3}}{b_{1}^{3}}
$$

其中 $a_{1}=E\left(\xi_{1}\right)$ (数学期望)， $b_{1}^{2}=D \xi_{1}$ (方差), $C$ 为一独立常数且 $\frac{1}{\sqrt{2 \pi}} \leqslant c<0.82$.

引理 2 对 $\forall x \in(0,1)$, 当 $n$ 足够大时, 有

$$
\left|\sum_{\substack{k \\ n+a(n) \\ k \leqslant n}} p_{n k}(x)-\frac{1}{2}\right| \leqslant \frac{2}{\sqrt{n x(1-x)}}+\frac{\alpha(n)}{\sqrt{n}} \sqrt{\frac{x}{1-x}},
$$

其中 $\alpha(n)$ 满足 (1.3) 式.

引理 3 对 $\forall x \in(0,1), 0 \leqslant k^{\prime} \leqslant n$, 有

$$
p_{n k^{\prime}}(x) \leqslant 5 / 2 \sqrt{n x(1-x)} \text {. }
$$

在叙述下面引理之前, 先引进一个记号, 令

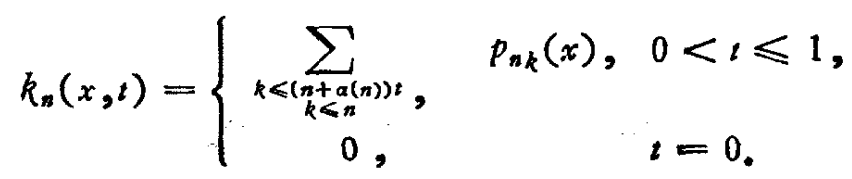

则有

$$
\begin{aligned}
& C_{n}(f, x)=\int_{0}^{1} f(t) d_{t} K_{n}(x, t), \\
& C_{n}^{*}(f, x)=\int_{0}^{1}\left(\varphi_{n}+1\right)\left(\int_{t \varphi_{n} /\left(1+\varphi_{n}\right)}^{\left(t \varphi_{n}+1\right) /\left(1+\varphi_{n}\right)} f(u) d u\right) d_{t} K_{n}(x, t) .
\end{aligned}
$$

对上述定义之 $k_{n}(x, t)$ 有以下不等式:

引理 4 当 $n$ 足够大时

(1) 若 $0 \leqslant y<x$, 有

$$
k_{n}^{\prime}(x, y) \leqslant \frac{x(1-x)}{n(x-y)^{2}}
$$

(2) 若 $x<z \leqslant 1$, 有

$$
1-k_{n}(x, z) \leqslant \frac{x(1-x)}{n(z-x)^{2}}
$$

\section{三、主要 定理}

定理 1 设 $f \in B V[0,1]$, 对 $\forall x \in(0,1)$, 当 $n$ 足够大时, 有

$$
\begin{aligned}
& \left|C_{n}(f, x)-\frac{1}{2}(f(x+)+f(x-))\right| \\
& \quad \leqslant \frac{3(x(1-x))^{-1}}{n} \sum_{k=1}^{n} \bigvee_{x=x / \sqrt{k}}^{x+(1-x) / \sqrt{k}}\left(g_{x}\right)+\frac{4+\alpha(n)}{\sqrt{n x(1-x)}}|f(x+)-f(x-)|,
\end{aligned}
$$

其中 $C_{n}(f, x)$ 为 $(1.1)$ 式所定义且满足 $(1.3)$ 式. $V_{a}^{b}\left(g_{x}\right)$ 表示 $g_{x}$ 在 $[a, b]$ 上的全变差. $g_{x}$ 定 义为 


$$
g_{x}(t)=\left\{\begin{array}{cl}
f(t)-f(x+), & x<t \leqslant 1 \\
0, & t=x ; \\
f(t)-f(x-), & 0 \leqslant t<x
\end{array}\right.
$$

证首先

$$
f(t)=\frac{1}{2}(f(x+)+f(x-))+g_{x}(t)+\frac{1}{2}(f(x+)-f(x-)) \operatorname{sgn}(t-x),
$$

因而

$$
\begin{aligned}
& \left|C_{n}(f, x)-\frac{1}{2}(f(x+)+f(x-))\right| \\
& \quad \leqslant\left|C_{n}\left(g_{x}(t), x\right)\right|+\frac{1}{2}|f(x+)-f(x-)|\left|C_{n}(\operatorname{sgn}(t-x), x)\right| .
\end{aligned}
$$

先估计 $C_{n}(\operatorname{sgn}(t-x), x)$.

若对 $\forall K>0, x \neq \frac{K}{n+\alpha(n)}$, 则

$$
C_{n}(\operatorname{sgn}(t-x), x)=\sum_{\frac{k}{n+a(n)}>x} p_{n k}(x)-\sum_{\frac{k}{n+a(n)}<x} p_{n k}(x) \stackrel{\text { def }}{=} A_{n}(x)-B_{n}(x) .
$$

注意到 $A_{n}(x)+B_{n}(x)=1$, 应用(2.2)式知

$$
\begin{aligned}
\left|C_{n}(\operatorname{sgn}(t-x), x)\right| & =\left|A_{n}(x)-B_{n}(x)\right| \\
= & \left|2 A_{n}(x)-1\right| \leqslant \frac{4}{\sqrt{n x(1-x)}}+\frac{2 \alpha(n)}{\sqrt{n}} \sqrt{\frac{x}{1-x}} .
\end{aligned}
$$

若对某 $k^{\prime}$, 有 $x=\frac{k^{\prime}}{n+\alpha(n)}$, 则

$$
A_{n}(x)+B_{n}(x)+p_{n k^{\prime}}(x)=1,
$$

而据引理 3 的 (2.3)式知

$$
\begin{aligned}
\left|C_{n}(\operatorname{sgn}(t-x), x)\right| & =\left|A_{n}(x)-B_{n}(x)\right| \\
& \leqslant\left|2 A_{n}(x)-1\right|+p_{n k^{\prime}}(x) \leqslant \frac{13}{2 \sqrt{n x(1-x)}}+\frac{2 \alpha(n)}{\sqrt{n}} \sqrt{\frac{x}{1-x}} .
\end{aligned}
$$

下面估计 $C_{n}\left(g_{x}(t), x\right)$, 为此, 将 $[0,1]$ 分为三部分:

$$
l_{1}=\left[0, x-\frac{x}{\sqrt{n}}\right], \quad I_{2}=\left[x-\frac{x}{\sqrt{n}}, x+\frac{1-x}{\sqrt{n}}\right], \quad I_{3}=\left[x+\frac{1-x}{\sqrt{n}}, 1\right] .
$$

应用 (2.5) 式, 有

$$
\begin{aligned}
C_{n}\left(g_{x}, x\right) & =\int_{0}^{1} g_{x}(t) d_{t} k_{n}(x, t)=\int_{I_{1}}+\int_{I_{2}}+\int_{I_{3}} \\
& \stackrel{\text { det }}{=} L_{n}(f, x)+M_{n}(f, x)+R_{n}(f, x) .
\end{aligned}
$$

应用和文献 [1]中完全相同的方法, 并结合应用引理 4 中的(2.7)、(2.8)式 (具体作法可参看文 献 [1], p. 270-274, 此不详述), 我们可以得到以下估计:

$$
\begin{aligned}
& \left|M_{n}(f, x)\right| \leqslant \bigvee_{x-x / \sqrt{n}}^{x+(1-x) / \sqrt{n}}\left(g_{x}\right), \\
& \left|L_{n}(f, x)\right| \leqslant \frac{2}{n x(1-x)} \sum_{k=1}^{n} \bigvee_{x-x / \sqrt{k}}^{x}\left(g_{x}\right),
\end{aligned}
$$

第 20 期

$$
\text { 科学 通报 }
$$




$$
\left|R_{n}(f, x)\right| \leqslant \frac{2}{n x(1-x)} \sum_{k=1}^{n} \bigvee_{x}^{x+(1-x) / \sqrt{x}}\left(g_{x}\right),
$$

由 (3.3)-(3.8) 式可知 (3.1) 式成立.

定理 2 设 $f \in B V[0,1]$, 对 $\forall x \in(0,1)$ ，当 $n$ 足够大时, 有

$$
\begin{aligned}
& \left|C_{n}^{*}(f, x)-\frac{1}{2}(f(x+)+f(x-))\right| \\
& \leqslant \frac{5}{2 \sqrt{n x(1-x)}} V_{0}^{1}(f)+\frac{3(x(1-x))^{-1}}{n} \sum_{k=1}^{n} \bigvee_{x-x / \sqrt{k}}^{x+(1-x) / \sqrt{k}}\left(g_{x}\right) \\
& \quad+\frac{5+\alpha(n)}{\sqrt{n x(1-x)}}|f(x+)-f(x-)|,
\end{aligned}
$$

其中 $C_{n}^{*}(f, x)$ 为 (1.4)式定义且满足 (1.3)式, $g_{x}(t)$ 为(3.2)式定义。

证 由于 $f$ 是 $[0,1]$ 上的有界变差函数, 故

$$
f(t)=\frac{1}{2}\left[\left(V_{0}^{t}(f)+f(t)\right)-\left(V_{0}^{t}(f)-f(t)\right)\right]
$$

记 $V_{0}^{t}(f)=F(t)$, 可知 $F(t)+f(t)$ 及 $F(t)-f(t)$ 皆为单增函数. 于是

$$
C_{n}^{*}(f, x)=\frac{1}{2}\left[C_{n}^{*}(F+f, x)-C_{n}^{*}(F-f, x)\right] \text {. }
$$

另外,

$$
C_{n}^{*}(F \pm f, x)=\sum_{k=0}^{n}\left(\varphi_{n}+1\right) \int_{k /\left(\varphi_{n}+1\right)}^{(k+1) /\left(\varphi_{n}+1\right)}(F(u) \pm f(u)) d u p_{n k}(x),
$$

由 $F+f$ 及 $F-f$ 的单增性, 可知

$$
\begin{gathered}
m_{1} \stackrel{\text { def }}{=} \sum_{k=0}^{n}\left(F\left(\frac{k}{\varphi_{n}+1}\right)+f\left(\frac{k}{\varphi_{n}+1}\right)\right) p_{n k}(x) \leqslant C_{n}^{*}(F+f, x) \\
\leqslant \sum_{k=0}^{n}\left(F\left(\frac{k+1}{\varphi_{n}+1}\right)+f\left(\frac{k+1}{\varphi_{n}+1}\right)\right) p_{n k}(x) \stackrel{\text { det }}{=} M_{1}, \\
m_{2} \stackrel{\text { det }}{=} \sum_{k=0}^{n}\left(F\left(\frac{k}{\varphi_{n}+1}\right)-f\left(\frac{k}{\varphi_{n}+1}\right)\right) p_{n k}(x) \leqslant C_{n}^{*}(F-f, x) \\
\leqslant \sum_{k=0}^{n}\left(F\left(\frac{k+1}{\varphi_{n}+1}\right)-f\left(\frac{k+1}{\varphi_{n}+1}\right)\right) p_{n k}(x) \stackrel{\text { def }}{=} M_{2},
\end{gathered}
$$

于是

$$
\frac{1}{2}\left(m_{1}-M_{2}\right) \leqslant C_{n}^{*}(f, x) \leqslant \frac{1}{2}\left(M_{1}-m_{2}\right) .
$$

从而

$$
\begin{aligned}
& \left|C_{n}^{*}(f, x)-\frac{1}{2}(f(x+)+f(x-))\right| \\
& \quad \leqslant \max \left\{\left|\frac{1}{2}\left(M_{1}-m_{2}\right)-\frac{1}{2}(f(x+)+f(x-))\right|,\right. \\
& \left.\left|\frac{1}{2}\left(m_{1}-M_{2}\right)-\frac{1}{2}(f(x+)+f(x-))\right|\right\} .
\end{aligned}
$$


而

$$
\begin{aligned}
M_{1}-m_{3}= & \sum_{k=0}^{n}\left[\left(F\left(\frac{k+1}{\varphi_{n}+1}\right)-F\left(\frac{k}{\varphi_{n}+1}\right)\right)+\left(f\left(\frac{k+1}{\varphi_{n}+1}\right)-f\left(\frac{k}{\varphi_{n}+1}\right)\right)\right] p_{n k}(x) \\
& +2 \sum_{k=0}^{n} f\left(\frac{k}{\varphi_{n}+1}\right) p_{n k}(x) .
\end{aligned}
$$

由引理 3 的 (2.3) 式知上式右端第一项的绝对值不超过 $\frac{5}{\sqrt{n x(1-x)}} V_{0}^{1}(f)$, 而由定理 1 知

$$
\begin{aligned}
& \left|\sum_{k=0}^{n} f\left(\frac{k}{\varphi_{n}+1}\right) p_{n_{k}}(x)-\frac{1}{2}(f(x+)+f(x-))\right| \\
& \quad \leqslant \frac{3(x(1-x))^{-1}}{n} \sum_{k=1}^{n} \bigvee_{x \rightarrow x / \sqrt{k}}^{x+(1-x) / \sqrt{k}}\left(g_{x}\right)+\frac{5+\alpha(n)}{\sqrt{n x(1-x)}}|f(x+)-f(x-)|,
\end{aligned}
$$

于是

$$
\begin{aligned}
& \left|\frac{1}{2}\left(M_{1}-m_{2}\right)-\frac{1}{2}(f(x+)+f(x-))\right| \\
& \leqslant \frac{5}{2 \sqrt{n x(1-x)}} V_{0}^{1}(f)+\frac{3(x(1-x))^{-1}}{n} \sum_{k=1}^{n} \bigvee_{x-x / \sqrt{k}}^{x+(1-x) / \sqrt{k}}\left(g_{x}\right) \\
& \quad+\frac{5+\alpha(n)}{\sqrt{n x(1-x)}}|f(x+)-f(x-)| .
\end{aligned}
$$

类似地可证

$$
\left|\frac{1}{2}\left(m_{1}-M_{2}\right)-\frac{1}{2}(f(x+)+f(x-))\right|
$$

也不超过上式右端的值. 因而由(3.11)式可证得定理的结论.

\section{四、注释}

(1) 定理 1 中, 若令 $\alpha(n)=0$, 则 $C_{n}(f, x)=B_{n}(f, x)$. 故由定理 1 可推得

$$
\begin{aligned}
\mid B_{n}(f, x) & -\frac{1}{2}(f(x+)+f(x-)) \mid \\
\leqslant & \frac{3(x(1-x))^{-1}}{n} \sum_{k=1}^{n} \bigvee_{x-x / \sqrt{k}}^{x+(1-x) / \sqrt{k}}\left(g_{x}\right) \\
& +\frac{4}{\sqrt{n x(1-x)}}|f(x+)-f(x-)|,
\end{aligned}
$$

此式显然改进了文献 [1] 中所得结果.

（2）定理 2 中, 若令 $\alpha(n)=0$, 则 $C_{n}^{*}(f, x)=K_{n}(f, x)$. 后者表示周知的 BernsteinKantorovitch 算子.

（3）我们说明定理 1 的阶是不能改进的. 考虑函数 $f(t)=|t-x|$. 由文献 [1] (2.6) 式知, 当 $n>\frac{2}{x(1-x)}$ 时,有 


$$
\frac{1}{16}(x(1-x))^{\frac{1}{2}} \leqslant B_{n}(|t-x|, x) \leqslant \frac{5}{2}\left(\frac{x(1-x)}{n}\right)^{\frac{1}{2}}
$$

而

$$
\begin{aligned}
& \left|C_{n}(|t-x|, x)-B_{n}(|t-x|, x)\right| \\
& \quad \leqslant \sum_{k=0}^{n}|| \frac{k}{n}-x|-| \frac{k}{n+\alpha(n)}-x|| p_{n k}(x) \\
& \quad \leqslant \sum_{k=0}^{n} \frac{\alpha(n) k}{n(n+\alpha(n))} p_{n k}(x)=\frac{\alpha(n)}{n+\alpha(n)} x=0\left(\frac{1}{\sqrt{n}}\right),
\end{aligned}
$$

上式最后等式用到条件 (1.3). 据 (4.1) 式知, 当 $n$ 足够大时, 有

$$
C_{n}(|\iota-x|, x) \geqslant \frac{1}{17}\left(\frac{x(1-x)}{n}\right)^{\frac{1}{2}} \text {. }
$$

而据 (3.1) 式, 知

$$
\begin{gathered}
\left|C_{n}(|t-x|, x)\right| \leqslant \frac{3(x(1-x))^{-1}}{n} \sum_{k=1}^{n} \bigvee_{x-x / \sqrt{k}}^{x+(1-x) / \sqrt{k}}\left(g_{x}\right) \\
\leqslant \frac{3(x(1-x))^{-1}}{n} \sum_{k=1}^{n} \frac{1}{\sqrt{k}} \leqslant \frac{3(x(1-x))^{-1}}{\sqrt{n}} .
\end{gathered}
$$

比较 (4.2) 及 (4.3) 式可以得到我们的结论.

（4）定理 2 的阶也是不能改进的.

\section{参考文献}

[1] Cheng Fuhua, J. Approx. Th., 39(1983), 3: 259-274.

[2]. Sikkema, P. C., Proc. Kon. Ned. Akad. Wetenschappen, Amsterdam, Auch in Indagationes Math., 78(1975), 243-253.

[ 3 ] 王仁宏, 无界函数逼近,科学出版社, 1983.

[ 4] 王样坤,概率论基础及其应用,科学出版社，1979. 